\title{
ACTITUD DE LOS PROFESORES HACIA LA INCLUSIÓN EDUCATIVA
}

\author{
Maribel GRANADA AZCÁRRAGA ${ }^{1}$ \\ María Pilar POMÉS CORREA \\ Susan SANHUEZA HENRÍQUEZ
}

\begin{abstract}
Resumen
La actitud del profesor es fundamental en el proceso de inclusión educativa, entendiendo por actitud un conjunto de percepciones, creencias, sentimientos a favor o en contra y formas de reaccionar ante la postura educativa que centra su esfuerzo en el logro de los aprendizajes de todos los estudiantes.

El propósito de este trabajo es describir analíticamente algunas dimensiones centrales que impactan en las actitudes que los profesores manifiestan hacia la inclusión educativa. Los factores que se describen son la experiencia de los docentes, las características de los estudiantes, el tiempo y recursos de apoyo, y la formación docente y capacitación. Cada uno de estos factores afecta las actitudes que los docentes puedan tener, limitando o facilitando sus intentos de generar prácticas más inclusivas. Mientras más factores afecten negativamente las prácticas pedagógicas de un profesor, menor será la probabilidad de que el docente manifieste una mejor actitud hacia la inclusión educativa.

El asumir una cultura, políticas y prácticas más inclusivas significará entregarle apoyos específicos, recursos ajustados, tiempo y espacios apropiados a los profesores para que ellos puedan servir a todos sus estudiantes con mayor efectividad y calidad.
\end{abstract}

Palabras clave: actitud, profesores, inclusión

\section{Resumé}

L'attitude du professeur est fondamentale dans le processus d'inclusion éducative, en prenant par attitude l'ensemble des perceptions, des croyances, des sentiments à faveur ou en contre et les

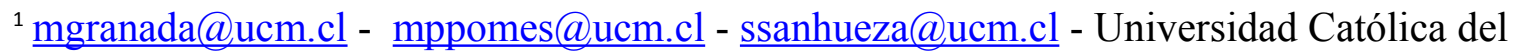

Maule, Chile

Fecha de recepción del artículo: Febrero 2013

Fecha de evaluación: Abril 2013 
façons de réagir devant la posture éducative que focalise l'effort dans la réussite des apprentissages de tous les étudiants.

Le but de ce travail est décrire analytiquement quelques dimensions centrales qui touchent dans les attitudes que les professeurs ont vers l'inclusion éducative. Les facteurs décrits sont l'expérience des professeurs, les caractéristiques des étudiants, le temps et les ressources d'appui, et la préparation du professeur et la formation. Chacun de ces facteurs touche les attitudes que les enseignants puissent avoir, limitant ou facilitant ses efforts pour générer pratiques plus inclusives. Plus il y a des facteurs qui touchent de façon négative les pratiques pédagogiques du maître, la probabilité de que lui aie une attitude meilleur vers l'inclusion éducative sera moindre.

Assumer une culture, politiques et pratiques plus inclusives signifiera donner des appuis spécifiques, ressources justes, temps et lieus appropriés aux enseignants pour qu'ils puissent servir avec plus d'efficacité et de qualité à tous les étudiants.

Mots-clés: Attitude, professeurs, inclusion

\begin{abstract}
Teachers' attitude is crucial for promoting inclusive education. Attitude can be defined as an array of perceptions, believes, feelings in favor or against someone or something; in addition, it is understood as ways to react to a specific educational approach that focuses its intention in the achievement of all students.

The purpose of this manuscript is to analytically describe central dimensions that impact on the attitude that teachers express towards inclusive education. Factors that are described in this work are teachers' experience, students' characteristics, time and resources of support, pre-service and inservice teacher training. Each one of these factors might impact teachers' attitude, minimizing or facilitating their attempts to generate more inclusive practices. Whereas more factors negatively impact teachers' pedagogical practices, it will be less likely that their attitude express a positive attitude towards inclusive education.

Embracing more inclusive culture, politics and practices means to provide specific support, adjusted resources, and appropriate settings for teachers in order to facilitate their role serving all their students with more effectiveness and high quality.
\end{abstract}

Key words: attitude, teachers, inclusion.

\title{
Introducción
}

El modelo inclusivo es hoy en día un referente para muchos contextos educativos que ven la necesidad de implementar estos principios al interior de sus comunidades educativas.

Dentro de este marco, existen muchos factores que están interviniendo en el éxito o fracaso de esta propuesta. Un elemento que emerge con relevancia es la actitud del profesorado hacia la inclusión educativa, pues ésta puede facilitar la implementación o puede constituirse en una barrera para el aprendizaje y la participación del alumnado.

En este sentido se hace necesario conceptualizar la inclusión educativa. Según los autores Booth y Ainscow (2002) es un conjunto de procesos orientados a eliminar o minimizar las barreras que limitan el aprendizaje y la participación de todos los estudiantes. Sus dimensiones incorporan la cultura, política y práctica. Dentro de la cultura se plantea una comunidad escolar con valores y creencias compartidos y orientados a que todos aprendan, implicando a la escuela en su conjunto; estudiantes, miembros del consejo escolar y familias. Las Políticas por su parte apuntan a focalizar a la inclusión como centro de desarrollo de la escuela y constituyen un único marco que orienta los distintos apoyos para responder a la diversidad. Así mismo, las Prácticas aseguran que las 
actividades escolares favorezcan la participación de todos evidenciando en coherencia la cultura a la que pertenecen y la política orientadora que poseen (Booth \& Ainscow, 2011).

El éxito de una mayor inclusividad educativa está marcado por ciertos aspectos relevantes que se relacionan según Eisenman, Pleet, Wandry y McGinley (2011), con el liderazgo de las escuelas, una cultura colaborativa, arreglos y adaptaciones de infraestructura que facilita el acceso, posibilidad de compartir la experiencia de otros profesores y con el desarrollo profesional de especialistas. A su vez, Idol (2006), plantea diversos indicadores del éxito de las prácticas inclusivas dentro de un contexto escolar, a saber:

-Los tipos de discapacidad de los estudiantes que participan en educación especial.

-La cantidad de tiempo que los estudiantes de educación especial utilizan aprendiendo en el programa de educación general de la escuela.

-La cantidad de personal de apoyo disponible y cómo ellos son utilizados.

-La cantidad y tipo de derivaciones para realizar evaluación especial

-La percepción de los miembros de la comunidad educativa sobre sus propias habilidades para generar cambios en sus prácticas pedagógicas y modificaciones curriculares, y además en sus habilidades para mantener la disciplina de los estudiantes y el manejo de la clase.

-La percepción de los miembros de la comunidad educativa sobre el impacto de las prácticas inclusivas en otros estudiantes.

Este autor señala como indicador de éxito de las prácticas inclusivas, la actitud de miembros de la comunidad educativa hacia otros miembros, hacia el trabajo colaborativo, hacia los estudiantes con necesidades educativas especiales (NEE) y hacia la inclusión.

Así se desprende que la actitud del profesor es fundamental en el proceso de inclusión educativa, entendiéndose por actitud una posición u orientación del pensamiento, que se traduce en una forma determinada de pensar, actuar o reaccionar (Beltrán, 1998) Este término "actitud" (postura) viene del italiano attitudine que es una deformación del latín aptitudini .Así mismo, se señala que es una disposición de ánimo de algún modo manifiesta.

La actitud se compone de tres dimensiones: a) la cognoscitiva, formada por percepciones, creencias e información que se tiene sobre algo. b) La afectiva que implica sentimientos a favor o en contra de algo y c) la conductual referida a la tendencia a reaccionar hacia algo de una cierta manera (Beltran, 1998)

Así entonces, en el tema de inclusión educativa, se concluye que la actitud es un conjunto de percepciones, creencias, sentimientos a favor o en contra y formas de reaccionar ante la postura educativa que centra su esfuerzo en el logro de los aprendizajes de todos los estudiantes.

El propósito de este escrito es describir analíticamente algunas dimensiones centrales que impactan en las actitudes que los profesores manifiestan hacia la inclusión educativa.

\section{Contexto de cambio}

Los cambios a nivel de políticas educacionales que están experimentando diferentes países, han influido mucho en el escenario en el que se desenvuelven los docentes tanto a nivel de educación común, como de educación especial, lo que ha implicado para ambos profesionales de la educación asumir nuevos roles que se fundamentan en la colaboración entre profesionales expertos en los contenidos de educación general y expertos en pedagogía de educación especial (Eisenman, Pleet, Wandry, McGinley, 2011).

Según Cardona (2006), distintas investigaciones sobre las actitudes de los profesores hacia la inclusión educativa, evidencian que ésta puede cambiar en función de un conjunto de variables referidas a la experiencia en el proceso educativo, las características de los alumnos, la disponibilidad de recursos, la formación, el apoyo y tiempo disponible. 
Investigaciones de los autores de Boer, Pijl \& Minnaert (2011), plantean que los profesores/as son personas claves en la implementación de la educación inclusiva. Una actitud positiva juega un rol esencial en la implementación de cambios educacionales exitosos. Estos autores dan cuenta de la revisión de 26 estudios que muestran en su totalidad que la mayoría de los profesores posee una actitud neutral o negativa hacia la inclusión de estudiantes con NEE en la educación regular, siendo la capacitación, género, años de experiencia trabajando en ambientes inclusivos y tipo de necesidades educativas, factores que impactarían en las actitudes de los profesores.

Por su parte, Jordan, Glenn, \& McGhie-Richmond (2010), señalan que para colaborar a que los sistemas escolares se transformen en instituciones inclusivas es necesario comprender la naturaleza de las creencias de los educadores y cómo estas creencias se relacionan con las acciones y prácticas profesionales. Asimismo, señalan que el liderazgo, las normas y cultura escolar pueden influir en las percepciones y creencias de los profesores acerca de la inclusión educativa.

\section{Factores que impactan en las actitudes de los docentes}

Los docentes en su práctica profesional no sólo deben dominar los contenidos que imparten sino, que es necesario que faciliten el aprendizaje y la participación de todos los estudiantes, propiciando oportunidades de mayor desarrollo e inclusión educativa (Sola, 1997). En la medida que el profesor asuma esta doble responsabilidad manifestará una actitud más positiva ante los estudiantes con distintas necesidades educativas.

Ahora bien, considerando que la actitud del profesor hacia la inclusión educativa está condicionada a la presencia de diferentes factores que pueden facilitar $u$ obstaculizar sus prácticas inclusivas, se hace necesario centrarse en estos aspectos, a saber: (a) La experiencia de los docentes, (b) Las características de los estudiantes, (c) El tiempo y recursos de apoyo, y (d) La formación docente y capacitación. A continuación se presenta una breve descripción de cada uno de ellos.

a) La Experiencia Docente:

La experiencia que poseen los profesores es entendida como el hecho de haber conocido, sentido o vivido una determinada práctica educativa, si esta práctica ha sido positiva o no y el tiempo de ejercicio profesional que ha implicado. Así, se ha señalado que los años de experiencia de los profesores influye en la actitud que se tiene hacia la educación inclusiva, los profesores con menos años de experiencia enseñando manifiestan una actitud más positiva que aquellos con más experiencia. Asimismo, se plantea que los profesores que tienen experiencias previas en educación inclusiva muestran una actitud más positiva que aquellos con menos experiencia en contextos inclusivos (de Boer et al., 2011).

En este sentido es posible precisar que la experiencia de los docentes puede influir en dos sentidos. La primera en cuanto a la cantidad de años de trabajo que un profesor tiene y la segunda en relación a experiencias previas vinculadas a las prácticas inclusivas. En el primer caso, más tiempo como docente no estaría favoreciendo la inclusión educativa, mientras que la experiencia específica en educación inclusiva impactaría positivamente su actitud, marcando la diferencia con aquellos docentes que no han experimentado instancias inclusivas en su quehacer pedagógico.

b) Las Características de los Estudiantes:

El concepto de necesidades educativas especiales (NEE), surge desde el Informe Warnock (1978), donde se centra la atención en el apoyo específico que requiere una persona en función de sus distintas necesidades que pueden ser permanentes o transitorias.

Las NEE permanentes, son aquellas dificultades que se presentan durante toda la trayectoria escolar $\mathrm{y}$ vida en general. Se encuentran las deficiencias visuales, auditivas, motoras e intelectuales entre otras. Las NEE transitorias son problemas de aprendizaje que se presentan durante un periodo 
escolar determinado, requiriendo una atención pedagógica específica (Ministerio de Educación de Chile [MINEDUC], 2009).

Parece ser que las características que presentan los estudiantes estarían condicionando las actitudes de los profesores ante el proceso de inclusión educativa. Así, Huang \& Diamond (2009), manifiestan que el tipo de discapacidad parece jugar un importante rol en la disposición de profesores y administrativos hacia la inclusión. Los estudios indican que los profesores responden de manera diferente según el tipo de discapacidad sin considerar el diagnóstico entregado. Así los profesores responden de manera más positiva hacia niños/as con discapacidades leves (Síndrome de Down), con discapacidad física (parálisis cerebral) y hacia aquellos niños/as sin un diagnóstico.

Por su parte, Cook \& Cameron, (2010) señalan que los estudiantes integrados con trastornos del aprendizaje y problemas conductuales reciben altos índices de rechazo desde sus profesores en relación a los estudiantes sin NEE. En tanto, estudiantes con problemas conductuales reciben mayores índices de rechazo que estudiantes con discapacidad intelectual. Asimismo, de Boer et al. (2011), sostienen que los educadores se muestran más negativos al hablar de inclusión de niños/as con trastornos de aprendizaje, problemas conductuales y discapacidad intelectual, que sobre la inclusión de estudiantes con discapacidad física o sensorial.

De acuerdo a lo planteado por los diferentes autores, los profesores tendrían una actitud diferente de acuerdo a las distintas necesidades que los estudiantes presentan, siendo los problemas conductuales los que concentran una respuesta más negativa, limitando las posibilidades de inclusión y participación de estos estudiantes. No se observa consenso en relación al impacto que causarían otras necesidades educativas como discapacidad intelectual, discapacidad física, discapacidad sensorial y dificultades específicas del aprendizaje.

Los estudiantes que no tienen un diagnóstico de NEE, serían menos rechazados que aquellos estudiantes con un diagnóstico. Al parecer aquellas discapacidades que generan mayor demanda del profesor recibirían los menores índices de aceptación.

c) Tiempo y recursos de apoyo

El tiempo disponible para enfrentar la inclusión educativa está referido a la posibilidad de contar con un lapso, espacio u oportunidad para realizar distintas acciones pedagógicas para abordar la tarea educativa como planificar, coordinar y colaborar.

Por su parte, Horne \& Timmons (2009) hacen notar que algunas de las preocupaciones de los profesores se relacionan con el tiempo disponible de planificación y el cubrir las necesidades de todos los estudiantes. En este sentido, el estudio en prensa de Sanhueza, Granada y Bravo (2011), establece que los recursos materiales y el tiempo continúan siendo percibidos por el profesorado como una dificultad para el desarrollo de prácticas inclusivas.

Mayores oportunidades para organizar el trabajo docente, podrían impactar en una actitud más positiva, abierta hacia la inclusión de estudiantes con NEE

Los recursos de apoyo, se entienden como los medios a través de los cuales se intenta dar respuestas educativas de calidad a las NEE. Dentro de estos elementos se puede distinguir, los recursos humanos y los recursos materiales.

En los recursos humanos se considera la participación de expertos en áreas específicas; profesionales, asistentes, compañeros y familia. Idol (2006), establece que el 77\% de los educadores indica que la mejor opción para educar a estudiantes con NEE es a través de la educación general siempre y cuando se cuente con todos los asistentes necesarios para trabajar con cada alumno que necesite apoyo.

En cuanto a los recursos materiales se consideran las adaptaciones curriculares y estrategias de enseñanza-aprendizaje. En este sentido Jordan, Glenn \& McGhie-Richmond (2010) indican que cuando las escuelas tienen acceso a una gran variedad de apoyos y a diversificadas estrategias de 
enseñanza, ellas pueden ser efectivas incluyendo a diversos estudiantes y manteniendo altos niveles de logros académicos.

Por su parte, estudios de Horne \& Timmons (2009), que confirman estudios previos en USA y Canadá, señalan que los profesores creen que necesitan clases con menos estudiantes, capacitación, tiempo de planificación, profesores asistentes de apoyo para realizar adaptaciones curriculares necesarias.

d) La Formación Docente y Capacitación.

Es sin duda importante que los profesores tengan una formación inicial profesional que les permita contar con herramientas para dar respuestas educativas de calidad a la diversidad de estudiantes en el proceso de enseñanza- aprendizaje. Y por otra parte, que cuenten con una formación profesional continua que permita capacitarse y actualizarse permanentemente, para responder a las distintas demandas emergentes. En este sentido diferentes estudios enfatizan la relevancia de la formación de profesores como un factor decisivo para hacer posible el proceso de inclusión educativa (Alegre 2000; Arnaiz 2003; Jiménez 2005 y Cardona 2006, citados en Sanchez, Díaz, Sanhueza y Friz, 2008; Stainback \& Stainback, 1999; Tilstone, Lani, \& Richard, 2003). Sin embargo, diversas investigaciones manifiestan más precisamente la necesidad de capacitación por parte de los profesores. En este sentido, Sanchez et al. (2008), sostienen que el 92\% de estudiantes de pedagogía, manifiesta que los profesores de educación regular no tienen la formación necesaria para atender a los alumnos con NEE.

En cuanto a la capacitación de los profesores en áreas determinadas para lograr una mayor calificación en el trabajo con estudiantes con NEE, los estudios de Woolfson \& Bradya (2009), señalan que resultados contradictorios han sido encontrados en investigaciones referidas a la relación entre capacitación y creencias de los profesores. Éstos se sienten poco preparados para enseñar en ambientes inclusivos. En este mismo sentido, de Boer \& Minnaert (2011) manifiestan que los profesores/as no se autocalifican como bien preparados para incluir estudiantes con discapacidad en sus aulas. En general los profesores no se sienten competentes ni a gusto enseñando a niños con diversas NEE. Ahora bien, si se compara a los profesores en términos de cantidad de capacitación, aquellos educadores que reciben más capacitación poseen una actitud más positiva que los profesores con menos capacitación.

Los profesores muestran preocupación ante su formación y sus capacidades de enfrentar prácticas más inclusivas (Lindsay, 2010). Por su parte, Horne \& Timmons (2009) enfatizan la importancia de contar con oportunidades de desarrollo profesional continuo, para así responder efectivamente al creciente número de necesidades especiales que presentan los estudiantes en la sala de clases, ya que establecen que los profesores se sienten insuficientemente preparados para cubrir las necesidades de sus estudiantes con NEE.

En este marco, Horne \& Timmons (2009) plantean la importancia de mantener una actitud positiva hacia la inclusión educativa, señalando que si no se ofrece un mayor apoyo administrativo, tiempo de planificación y capacitaciones sobre estrategias pedagógicas específicas según discapacidades, la percepción positiva que los profesores tienen se podría ver deteriorada.

\section{Conclusiones}

La generación de un proceso más inclusivo requiere de tiempo necesario, para integrar y asumir los cambios que demandan la implementación de un sistema abierto a la diversidad. Esto implica llevar a cabo las transformaciones necesarias, que se originan desde la creación de políticas inclusivas, desde una cultura que acoge y se hace cargo de la diversidad con prácticas pedagógicas, que responden a las distintas formas de aprender al interior de la comunidad escolar. 
La inclusión educativa presenta una complejidad que puede ser comprendida de mejor manera si se tiene atención sobre el profesor como agente relevante y clave de este proceso. Puede constituirse en una barrera o en un agente facilitador de las prácticas inclusivas.

Las actitudes del profesor acerca de la inclusión educativa, entendidas como el conjunto de percepciones, creencias, sentimientos y formas de actuar, impactan la disposición hacia la inclusión de personas con NEE. Una actitud positiva hacia prácticas inclusivas va a favorecer dicho proceso. En tanto una actitud negativa minimizará las oportunidades de aprendizaje y participación de estudiantes con algún tipo especial de necesidades educativas.

Los distintos factores que influyen en las actitudes de los profesores hacia la inclusión educativa, están referidos a variados aspectos:

La experiencia del docente en el proceso de enseñanza-aprendizaje. En este sentido, la cantidad de años de servicio que un profesor tiene no es garantía de una actitud más favorable hacia la inclusión educativa. Sin embargo, la experiencia previa en la atención a la diversidad si se visualiza como un factor que impacta positivamente en la actitud del docente hacia prácticas más inclusivas. La experiencia previa le ayudaría a dar respuestas educativas más ajustadas a los requerimientos, bajando la ansiedad frente a lo nuevo, dándole la oportunidad de replicar y optimizar algo que ya se ha hecho.

Las características de los estudiantes. Las necesidades educativas especiales que presentan los alumnos se constituyen en un factor que impacta la actitud que el profesor manifiesta hacia la inclusión. Sin embargo, estas necesidades se abordan de manera diferente, causando variados niveles de aceptación o rechazo en el profesorado. Las necesidades que alcanzan un mayor consenso en cuanto al menor nivel de aceptación son las dificultades conductuales. Las que demandarían de parte del profesor una mayor atención y recursos psicopedagógicos específicos para atenderlas y requerirían un mayor esfuerzo para mantener un clima adecuado al interior del aula que propicie el aprendizaje de todos los estudiantes.

Las necesidades referidas a la discapacidad intelectual leve, discapacidad física y sensorial presentarían un menor índice de rechazo por parte de los profesores, al igual que frente a los estudiantes con diagnósticos no conocidos. Esto posiblemente demanda de parte del profesor menos adaptaciones y menos esfuerzos pedagógicos para contener a los estudiantes con estas necesidades dentro del grupo curso.

El tiempo del que se dispone para implementar acciones educativas, se constituiría en otro factor relevante que impactaría la actitud del profesorado. Planificar, colaborar y coordinar el trabajo para atender las necesidades educativas especiales sin lugar a dudas requiere de momentos suficientes que le permitan a los docentes concretar estas acciones para dar respuestas educativas de alta calidad y que tengan un mayor impacto. El tiempo insuficiente continúa siendo una barrera para las prácticas más inclusivas.

Por su parte, los recursos con que se cuenta para facilitar el progreso del estudiante también podrían facilitar o limitar las posibilidades u oportunidades de generar espacios educativos más inclusivos. Cuando la unidad educativa cuenta con expertos en áreas específicas, asistentes y la colaboración de la familia se potencia más el aprendizaje y la participación de todos los estudiantes. En este contexto, el profesor se sentiría respaldado en la toma de decisiones, en la distribución de tareas, generación e implementación de sus prácticas, optimizando de esta manera su trabajo.

$\mathrm{Si}$ el profesor cuenta con la posibilidad de generar adaptaciones curriculares y estrategias diversificadas de enseñanza y aprendizaje, es posible sostener que su disposición frente a la atención a la diversidad será más positiva. En la medida en que la respuesta pedagógica del docente sea ajustada a las necesidades de sus estudiantes, existirán mayores probabilidades de que los alumnos tengan éxito y alcancen los objetivos pedagógicos propuestos, impactando positivamente la actitud del profesor hacia el proceso de aprendizaje de las personas con NEE. 
La formación profesional inicial del docente, que proporcione competencias para saber, hacer y ser en el ejercicio de la práctica docente, es una condición necesaria para responder a la diversidad. Los profesores de educación regular no se sienten preparados para asumir esta tarea ya que no cuentan desde su formación con las competencias necesarias para trabajar en ambientes educativos más inclusivos. Por tanto, la actitud de los docentes se ve impactada profundamente, limitando su disposición para acoger a los estudiantes con NEE.

La capacitación permanente de los profesores para responder a los desafíos pedagógicos es otro factor que influye en las actitudes hacia la inclusión educativa. La falta de capacitación se manifiesta en los docentes en un sentimiento de desagrado en la enseñanza a niños con NEE. En la medida que los docentes sean capacitados tendrán una actitud más positiva hacia la inclusión educativa.

Cada uno de estos factores descritos anteriormente afecta las actitudes que los docentes puedan tener, limitando o facilitando sus intentos de generar prácticas más inclusivas. Además habría que considerar que posiblemente la presencia de más de uno de estos factores podría hacer más complejo el abordaje de esta problemática. Mientras más factores afecten negativamente las prácticas pedagógicas de un profesor menos será la probabilidad de que el docente manifieste una mejor actitud hacia la inclusión educativa.

En este marco, y dada la complejidad que experimentan los docentes, en su quehacer pedagógico es posible pensar que son varios los factores que estarían gravitando e impactando en su disposición para enfrentar las nuevas demandas impuestas por un enfoque educativo más inclusivo. Un profesor con insuficiente formación inicial y capacitación, que posee poco tiempo para planificar su trabajo, sin experiencias previas atendiendo la diversidad tendrá mayor probabilidad de tener una actitud negativa.

Si la escuela quiere optar por un enfoque más inclusivo, que ofrezca mejores oportunidades de desarrollo y participación a todos los estudiantes, tendrá que en su conjunto comprometerse y hacerse cargo de cada uno de sus miembros, funcionando de un modo diferente, innovando en sus políticas, cultura y práctica al interior de la comunidad para sostener esta nueva manera de enfrentar el proceso educativo con mayor calidad. Este cambio debiese impactar positivamente la actitud del profesorado encargado de interactuar directamente con todos los estudiantes que forman parte de la escuela.

\section{BIBLIOGRAFÍA}

ALEGRE, M. (2000). Diversidad humana y educación. Málaga: Aljibe

ARNAIZ, P. (2003). Educación inclusiva: Una escuela para todos. Málaga: Ediciones Aljibe.

BELTRÁN, J. (1998). Procesos, estrategias y técnicas de aprendizaje. Madrid: Síntesis

BOOTH, T. y AINSCOW, M. (2002). Index for inclusion: Developing learning and participation in schools. Bristol: CSIE.

BOOTH, T. \& AINSCOW, M. (2011).Guía para la Inclusión Educativa. Reino Unido: CSIE

CARDONA, M. C. (2006). Diversidad y educación inclusiva: enfoques metodológicos y estrategias para una enseñanza colaborativa. Madrid: Pearson-PrenticeHall.

COOK, B. G. \& CAMERON, D. L. (2010). Inclusive teachers' concern and rejection toward their students. Investigating the validity of ratings and comparing student groups. Remedial and Special Education, 31, 67-76.

DE BOER, A., PIJL, S. J., \& MINNAERT, A. (2011). Regular primary schoolteachers' attitudes towards inclusive education: A review of the literature. International Journal of Inclusive Education, 15, 331-353. 
EISENMAN, L. T., PLEET, A. M., WANDRY, D., \& MCGINLEY, V. (2011).Voices of special education teachers in an inclusive high school: Redefining responsibilities. Remedial and Special Education 32, 91-104.

HORNE, P. E. \& TIMMONS, V. (2009). Making it work: Teachers' perspectives on inclusion. International Journal of Inclusive Education, 13, 273-28.

HUANG, H. \& DIAMOND, K. E. (2009). Early childhood teachers' ideas about including children with disabilities in programmes designed for typically developing children. International Journal of Disability, Development and Education, 56, 169-182.

IDOL, L. (2006).Toward Inclusion of Special Education Students in General Education. A Program Evaluation of Eight Schools. Remedial and Special Education, 27, 77-94.

JORDAN, A., GLENN, C., \& MCGHIE-RICHMOND, D. (2010). The Supporting Effective Teaching (SET) project: The relationship of inclusive teaching practices to teachers' beliefs about disability and ability, and about their roles as teachers. Teaching and Teacher Education, 26, 259-266

JORDAN, A., SCHWARTZ, E., \& MCGHIE-RICHMOND, D. (2009). Preparing teachers for inclusive classrooms. Teaching and Teacher Education 25, 535-542.

LINDSAY, G. (2010). Intervención en el lenguaje en una escuela inclusiva. En V. M. Acosta \& A.M. Moreno (Eds.), Dificultades del lenguaje, colaboración e inclusión educativa (pp. 93104). Barcelona: Lexus.

Ministerio de Educación de Chile. (2009).Decreto 170. Fija normas para determinar los alumnos con necesidades educativas especiales que serán beneficiarios de las subvenciones para educación especial. Gobierno de Chile.

SÁNCHEZ, A., DÍAZ, C., SANHUEZA, S. y FRIZ, M. (2008). Percepciones y actitudes de los estudiantes de pedagogía hacia la inclusión educativa. Estudios pedagógicos, XXXIV, № 2 : 169-178.

SANHUEZA, S., GRANADA, M. y BRAVO, L (en prensa) Actitudes del profesorado de Chile y Costa Rica hacia la inclusión educativa. Revista Cadernos de Pesquisa.

STAINBACK, W. And STAINBACK, S. (1999). Aulas inclusivas. Madrid: Edit. Narcea

SOLA, T. (1997). La formación inicial y su incidencia en la educación especial. En Sánchez Palomino, A. Y J. Torres González, Educación especial I. Una perspectiva curricular, organizativa y profesional. Madrid: Pirámide.

TILSTONE, CH., LANI, F. Y RICHARD, R. (2003). Promoción y desarrollo de prácticas educativas inclusivas. Madrid: Eos

WARNOCK REPORT (1978) Special Educational Needs. Report of the Committee of Enquiry into the Educational of handicapped Children and Young People. Laondon. Her Majesty's Stationery Office.

WOOLFSON, M. L. \& BRADY, K. (2009). An investigation of factors impacting on mainstream teachers' beliefs about teaching students with learning difficulties. Educational Psychology, 29,221-238. 